\title{
THE GEOMETRY OF MOVEMENT.
}

Geometrie der Bewegung in synthetischer Darstellung. Von Dr. Arthur Schoenflies. Leipzig, B. G. Teubner, $1886^{\circ}$. 8vo, pp. vi +194 .

La Géométrie du Mouvement. Exposé synthétique. Translated by Ch. Speckel, Capitaine du Génie. Paris, GauthierVillars, 1893. 8vo, pp. vii +292 .

Perhaps apology is needed for noticing a book no longer in its infancy. But we feel that "better late than never", applies to acquaintance with a work which contains so much matter which was new at the time of writing and is not yet accessible in English, nor (we believe) well known.

The main idea of the book is to consider a body in two or more positions relatively to another body, and thence as a limit case to discuss the instantaneous motion. Full advantage is taken of the duality arising from viewing things from the standpoint of the one body or the other. That we have found the book difficult is probably due to our early training; but a few more figures and a few more details would have been welcome.

The French translation is very reliable, and its value is increased by a good elementary account of complexes and congruences of lines (pp. 219-291). by G. Fouret.

Our intention is, not to discuss the information in the book, but to select a few of the more salient theorems (omitting such as are presumably familiar). When in this string of enunciations there seems occasion to interpolate a remark, square brackets are used.

\section{§1. Motion of a Plane in a Plane.}

(1) Let $\sigma_{0}, \sigma_{1}, \sigma_{2}$ be three positions of a plane $\sigma$, relative to a plane $\sigma^{\prime}$. Let $A_{0}, A_{1}, A_{2}$ be the three positions of a point $A$ of $\sigma, A^{\prime}$ the point of $\sigma^{\prime}$ equidistant from $A_{0}, A_{1}, A_{2}$. The correspondence of the points $A$ and $A^{\prime}$ is quadratic; to a line in the one plane corresponds a conic in the other, passing through the three centres of rotation in that other.

(2) All the lines of $\sigma$ which for the three positions meet in a point of $\sigma^{\prime}$ form a pencil in $\sigma$. All the points of $\sigma$ which lie on a line of $\sigma^{\prime}$ lie on the circumcircle of the centres of rotation in $\sigma$.

This circle is called proleptically the " circle of inflexions."

[Two questions arise here which are not considered in the book: What is the vertex of the pencil in $\sigma$, and what is 
the envelope of the lines in $\sigma^{\prime}$ ? To answer these consider the accompanying kaleidoscopic figure.

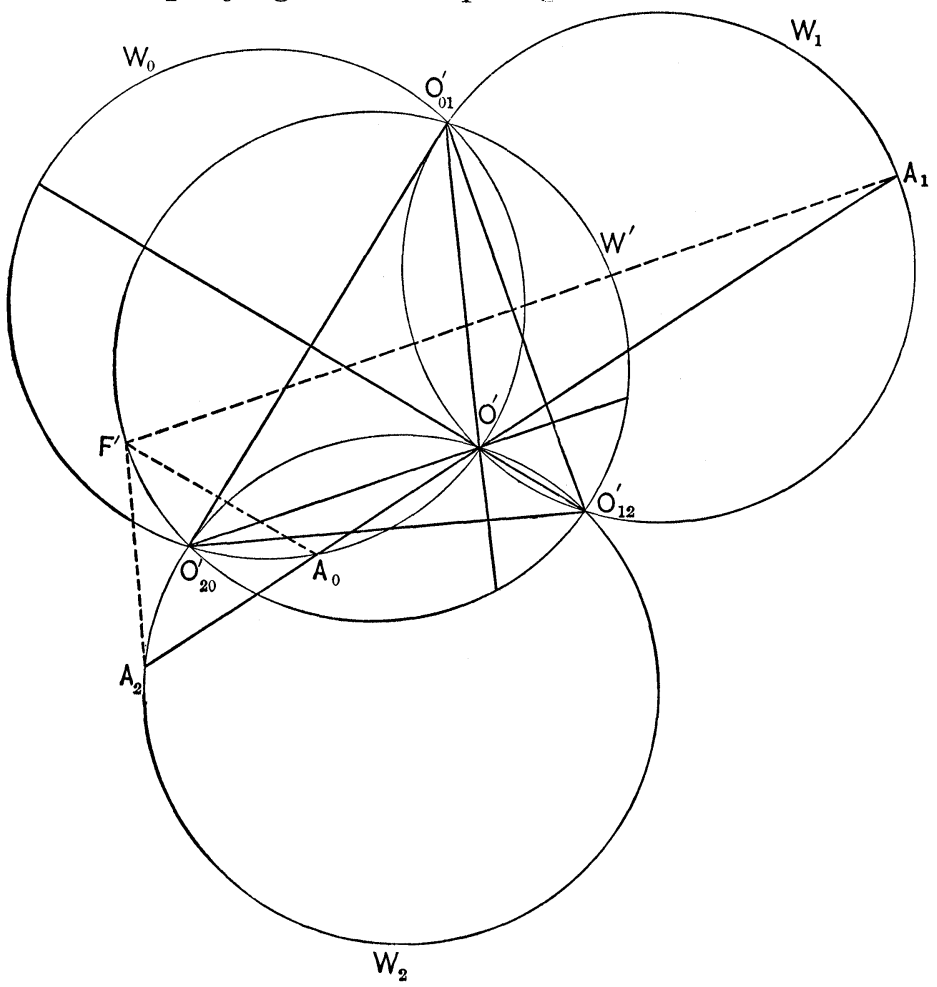

In the figure $O_{01}{ }^{\prime}, O_{12}{ }^{\prime}, O_{20}{ }^{\prime}$, are the centres of rotation about which $\sigma$ is brought from the position $\sigma_{0}$ into $\sigma_{1}, \sigma_{1}$ into $\sigma_{2}, \sigma_{2}$ into $\sigma_{0} . \quad O^{\prime}$ is the orthocentre of the three centres of rotation, and the four equal circles are the circles of inflexion. We may replace the rotations by pairs of reflexions ; $\sigma_{0}, \sigma_{1}$, and $\sigma_{2}$ are simply reflexions of $\sigma^{\prime}$ in the sides of the triangle.

The reflexions of a point $F^{\prime}$ on the circle $W^{\prime}$ lie on a line (the directrix of the inscribed parabola whose focus is $F^{\prime}$ ) which passes through $O^{\prime}$. Hence :

When a point of $\sigma$, in its three positions, lies on a line of $\sigma^{\prime}$, that line passes through the orthocentre $O^{\prime}$.

And hence, considering the inverse motion, when a line of $\sigma^{\prime}$, in three positions, passes through a point of $\sigma$, the line must pass through the orthocentre of the triangle of rotations in $\sigma^{\prime}$.

*Elsewhere also in the book the notion of reflexion might have been useful. 
These facts are implicitly contained in the theory of similar figures, as given in Casey's Analytic Geometry (second edition, p. 395) or in the same writer's Sequel to Euclid. But the kinematical importance of the case when the similar figures are equal is not pointed out by Casey.]

(3) For the instantaneous motion of $\sigma$ in $\sigma^{\prime}$, it is deduced that the points of inflexion lie at any instant on a circle; that the inflexional tangents all pass through a point; that the lines of $\sigma$ which are cuspidal tangents of their envelopes meet in a point, and that the locus of cusps is a circle. This circle of cusps for $\sigma$ is the circle of inflexions for $\sigma^{\prime}$.

[It is strange that this complete and satisfactory statement of the case should have escaped mention even in such books as Clifford's Kinematic. The identity of the circle of cusps with the circle of inflexions of the inverse motion was noticed by Aronhold.]

(4) Taking now four positions $\sigma_{0}, \sigma_{1}, \sigma_{2}, \sigma_{3}$ of $\sigma$, the point $A$, whose four positions lie on a circle of $\sigma^{\prime}$, must lie on a circular cubic curve, containing the six centres of rotation. The centres of the circles form a circular cubic in $\sigma^{\prime}$. In the inverse motion the curves interchange their rôles.

[More details on this cubic would be acceptable. And the remarkable figure formed by the six centres of rotation also calls for comment. Because the circles $W_{123}, W_{230}, W_{301}$, $W_{012}$ meet at a point, the pairs $O_{01}, O_{23} ; O_{02}, O_{31} ; O_{03}, O_{12}$ are in involution, but they satisfy a further condition ; for the orthocentres of the triangles 123 etc., lie on a line. The theory of similar figures would again be useful in this discussion ; but it must be observed that it can be applied in eight different ways.]

(5) In five positions of $\sigma$ there are in general 4 points which in the 5 positions lie on a circle.

\section{\$2. Motion of a Body about a fixed Point.}

(1) In two positions $S_{0}$ and $S_{1}$ in a space $S^{\prime}$ corresponding planes of two pencils meet on an orthogonal cone $(i$. e., on a cone of which a circular section is perpendicular to a generator).

Hence, in the instantaneous motion, the planes of a pencil touch their envelopes in lines which form an orthogonal cone. One set of circular sections is perpendicular to the axis of the pencil, the other to the instantaneous axis.

For three positions we have, as before, a quadratic correspondence between a moving ray $a$ and the axis of the circular cone of $S^{\prime}$ through the 3 positions of $a$. 
(2) Rays which in the 3 positions lie in a plane of $S^{\prime}$ form in $S$ a cubic cone containing the 3 instantaneous axes and the 6 lines of contact of tangents from these to the absolute cone.

Inversely, planes which in the 3 positions meet in a line of $S^{\prime \prime}$ touch a cone of the third class, the reciprocal of the other.

[Stripped of kinematical notions, these cones are the locus of a point whose reflexions in 3 planes which meet at $O$ lie in a plane through $O$, and the envelope of that plane.]

(3) For 4 positions, there are in general 6 rays $a$ for which $a_{0}, a_{1}, a_{2}, a_{3}$ lie in a plane.

[It will be noticed that cones are used throughout these theorems in preference to their intersections with a concentric sphere or with the plane at infinity.]

\section{§3. Motion of a Body $\Sigma$ in Space $\Sigma^{\prime \prime}$.}

(1) In two positions, the middle points of the two positions of points of a line $g$ of $\Sigma$ lie on a line $g^{m}$ of $\Sigma^{\prime}$.

(2) The displacement of the line can be effected by a rotation about a line $g^{\nu}$.

(3) The middle points of points of a plane of $\Sigma$ lie in a plane of $\Sigma^{\prime}$. tem.

(4) The lines $g^{m}$ and $g^{\nu}$ are conjugate lines of a null-sys-

(5) The most general motion of a rigid body is the rolling and sliding of a ruled surface on another. The parameters, for the generators in contact, must be the same.

[We are not repeating a well known proposition here, for the last condition is not brought ont clearly as a rule.]

(6) The tangents of the trajectories of all the points of a body, at any instant, form a tetrahedral complex. The fundamental tetrahedron reduces to the axis of rotation and the line at infinity in a perpendicular plane.

(7) The characteristics of a pencil of planes form at each instant, in general, an orthogonal hyperboloid ( $i$. e., a hyperboloid of which a set of circular sections is perpendicular to a generator).

(8) The lines of the complex which pass through a point form an orthogonal cone.

(9) All points of $\Sigma^{\prime}$ which are aiming at a point of $\Sigma^{\prime}$ lie on a circular cubic curve in space, the partial intersection of a right cylinder and an orthogonal cone. The axis of rotation is the real asymptote of the cubic.

[This pretty cubic can be very simply constructed by 
drawing the curve $y=\tan \frac{x}{2}$ and rolling the paper into a cylinder of radius 1 , so that the asymptotes coincide.]

(10) The characteristics of all planes which pass through a point are at any instant secants of a circular cubic space curve.

(11) All points of $\Sigma$ which, in three positions, lie in a line of $\Sigma^{\prime}$ form a cubic space curve through the points at infinity on the 3 axes of displacement.

(12) All points of $\Sigma$ which in 4 positions lie in a plane of $\Sigma^{\prime}$ form a cubic surface.

(13) All points of $\Sigma$ which in 5 positions lie in a plane form a sextic space curve.

(14) There are in general 10 points which in 6 positions lie in a plane.

(15) The points which in 5 positions lie on a sphere form a quartic surface. The centres of the spheres form a quartic in $\Sigma^{\prime}$. In the inverse movement the quartics interchange their rôles.

(16) The points which in 6 positions lie on a sphere form a space curve of order 10 .

(17) There are in general 20 points which in 7 positions lie on a sphere.

Haverford College.

F. Morley.

\section{THE NEW EDITION OF WEBER'S ALGEBRA.}

Lehrbuch der Algebra. By Heinrich Weber. Second edition. Braunschweig, Vieweg und Sohn, 1898-99. 8vo. Vol. I, pp. 703 . Vol. II, pp. 855 .

Most of the readers of the BuLletin have been aware that a new edition of Weber's Algebra was in progress. Some time ago the first volume appeared ; the work is now complete, the second volume of the new edition having just come out. The many excellencies of this great work have been so generally appreciated that Professor Weler has experienced the very unusual pleasure of seeing a new edition required in less than two years after the publication of the first.

The first question that those who already have purchased the first edition will wish answered is: Are the changes so great as to make it desirable to secure the new edition? To 